\title{
Experimental validation of the discrete-time MCS adaptive strategy
}

\author{
Mario di Bernardo ${ }^{\mathrm{b}, \mathrm{c}}$, Alessandro di Gaeta ${ }^{\mathrm{a}}$, Umberto Montanaro ${ }^{\mathrm{a}, *}$, Josep M. Olm ${ }^{\mathrm{d}}$, \\ Stefania Santini ${ }^{\mathrm{b}}$ \\ a Istituto Motori, National Research Council, 80125 Napoli, Italy \\ b Department of Electrical Engineering and Information Technology, University of Naples Federico II, 80125 Napoli, Italy \\ ${ }^{\mathrm{c}}$ Department of Engineering Mathematics, University of Bristol, Bristol BS8 1TR, UK \\ d Department of Applied Mathematics IV, Universitat Politècnica de Catalunya, 08860 Castelldefels, Spain
}

\section{A R T I C L E I N F O}

\section{Article history:}

Received 21 June 2011

Accepted 10 December 2012

Available online 23 March 2013

Keywords:

Model reference adaptive control

Discrete time systems

Validation

Adaptive systems

Adaptive algorithms

\begin{abstract}
A B S T R A C T
This paper is concerned with the implementation and experimental validation of a discrete-time model reference adaptive control strategy, known as Minimal Control Synthesis (MCS) algorithm. After discussing the proof of stability of the algorithm when applied to discretized models of continuous-time plants, the problem of controlling a highly nonlinear electro-mechanical device is taken as a representative case of study. It is shown that the discrete-time MCS is an effective strategy to solve the problem while guaranteeing robustness to unmodeled nonlinear dynamics over a wide range of test manoeuvres.
\end{abstract}

(c) 2013 Elsevier Ltd. All rights reserved.

\section{Introduction}

Model Reference Adaptive Control (MRAC) strategies are particularly useful in a wide range of applications, specifically those affected by noise and uncertainties. The Minimal Control Synthesis (MCS) algorithm is a type of MRAC strategy, first proposed in Stoten and Benchoubane (1990a, 1990b), that relaxes the original assumptions required by the classical approach proposed by Landau (1979). Essentially, in earlier MRAC controllers the control action includes a linear term, which is synthesized from the nominal values of the plant parameters, and has its origin in the non-null initial conditions assigned to the adaptive gains. Instead, the MCS does not require any a priori knowledge of the plant model with the adaptive gains being started from zero. Over the past few years, MCS controllers have been effectively used in a number of control problems, including chaotic systems, shaking tables in earthquake engineering, hydraulic pumps and robotic arms, e.g. Stoten and di Bernardo (1996), Stoten and Gómez (2001), Gizatullin and Edge (2007), di Bernardo, di Gaeta, Giglio, Montanaro, and Santini (2009), di Bernardo, di Gaeta, Montanaro, and Santini (2010), di Bernardo et al. (2010), Rossi, Irace, Montanaro, di Bernardo and Breglio (2010), and Montanaro, di Gaeta, and Giglio (2011). From a theoretical viewpoint, extensions to the original strategy and proof of asymptotic stability have been proposed, the most recent being hybrid MCS strategies in di Bernardo, Montanaro, and Santini (2007) and an extended MCS strategy for

\footnotetext{
* Corresponding author.

E-mail address: u.montanaro@im.cnr.it (U. Montanaro).
}

piecewise-affine systems in di Bernardo, Montanaro, and Santini (2010b), di Bernardo, Montanaro, and Santini (2008), di Bernardo, Hoyos Velasco, Montanaro, and Santini (2012), di Bernardo, Montanaro, and Santini (2013), and di Bernardo, Montanaro, Olm, and Santini (in press). Identification algorithms for piecewise-linear systems based on the adaptive gains of the MCS strategy can be found in di Bernardo, Montanaro, and Santini (2009) and di Bernardo, Montanaro, and Santini (2010a).

The MCS was originally developed for continuous-time plants although digital implementations based on a discretized MCS controller were proposed in Stoten and Benchoubane (1990a). Despite being validated experimentally and through simulations, no proof of asympotic stability was given for the discrete-time MCS. Indeed, to the authors' knowledge the first local stability analysis of a control system including a discrete-time MCS algorithm was reported in Bursi, Stoten, and Vulcan (2007): the study considered a first order system and linearization techniques were used. Notice also that the discretization of the plant and the MCS controller yields a significant reduction of the stability regions and a loss of dynamic performance, as recently shown in Bursi, Stoten, Tondini, and Vulcan (2009).

A formal proof of asymptotic stability for the discrete-time MCS algorithm was presented only very recently in di Bernardo, di Gennaro, Olm, and Santini (2010) for generic n-dimensional, discrete-time plants. As for its continuous-time counterpart, discrete-time MCS controllers are shown to be robust in the presence of parameter uncertainties, slow parameter variations with respect to the adaptation rate of the control gains - and matched external disturbances. 
A key assumption of the MCS algorithm is for the plant and reference model to be given in control canonical form. Often in applications, this is not the case; for example, when MCS is applied to continuous plants via digital microcontrollers. Indeed, in this case the plant has to be discretized, and the resulting matrices are not in canonical form with any of the usual discretization methods (see, for example Rugh, 1996).

The main objective of this paper is to show that discrete-time MCS controllers can be effectively used even in continuous-time plants discretized via a forward Euler's method, regardless of the fact that the discretized plant and reference model matrices are not in control canonical form.

When dealing with the synthesis of novel control strategies, the experimental analysis is a fundamental and crucial step of the design process to test the robustness and the performance of the closed-loop behavior in practical circumstances. In this work, the Electronic Throttle Body (ETB) system - which is an essential device dedicated to the regulation of the air mass flow rate in internal combustion engines (see di Bernardo, di Gaeta, Montanaro, et al., 2010 and references therein for further details) - is taken as a representative plant. Experimental results presented in the paper show the effectiveness of the discrete-time MCS algorithm in controlling this highly nonlinear electromechanical plant without requiring any precise experimental characterization or an accurate model of the system. It is worth emphasizing that the design of novel control approaches for the ETB is beyond the scope of this paper, which instead uses the ETB device as a challenging representative example to test the performance of the discrete-time MCS. A preliminary numerical validation of the technique was given in di Bernardo, di Gaeta, Montanaro, Olm, and Santini (2011).

The rest of the paper is organized as follows. The MCS strategy for discretized continuous-time systems is discussed in Section 2. Then, Section 3 contains a basic description of the Electronic Throttle Body being used, while Section 4 includes some details on the design of an MCS controller for the ETB, a description of the experimental setup and the experimental results. Concluding comments and suggestions for future work are outlined in Section 5. Appendix A provides details of the proof of the main result, which is stated in Section 2, and Appendix B presents a mathematical model of the ETB showing that it fits within the class of plants studied in Section 2 .

\section{MCS approach for discretized continuous-time plants}

Consider the continuous-time plant

$\dot{x}=A(t) x+B b u$

where $x=\left[x_{1}, \ldots x_{n}\right]^{T}$ is the state vector, $u$ is the system input, $b \in \mathbb{R} \backslash\{0\}$ and $A(t), B$ are, respectively, an $n \times n$ time-varying real matrix and $n$-dimensional vector assumed to be in control canonical form:

$A(t)=\left(\begin{array}{cccc}0 & 1 & \ldots & 0 \\ 0 & 0 & \ldots & 0 \\ \vdots & \vdots & \vdots & \vdots \\ 0 & 0 & \ldots & 1 \\ -a_{1}(t) & -a_{2}(t) & \ldots & -a_{n}(t)\end{array}\right), \quad B=\left(\begin{array}{c}0 \\ \vdots \\ 0 \\ 1\end{array}\right)$.

Canonical transformations to recast a given piecewise linear continuous system into this form are given in di Bernardo, Montanaro, and Santini (2011).

According to Stoten and Benchoubane (1990b), model (1) may include any external disturbance, plant nonlinearity and/or unmodelled term when they can be expressed as a matched uncertainty and described as an unknown variation in $A(t)$, preserving its canonical structure. Moreover, it is worth remarking that plant parameters are completely unknown: the procedure only requires knowledge of the sign of $b$, and $a_{j}(k)=a_{j}\left(t_{k}\right)$, $j=1, \ldots, n$, with $t_{k}$ being the generic sample instant, to be slowly varying with respect to the evolution of some adaptive gains of the control law that will be defined further on.

Assume that the reference model is given in the form:

$\dot{x}_{m}=A_{m} x+B b_{m} r$,

where $A_{m}$ is, again, an $n \times n$ real matrix with the same canonical structure as $A(t)$. The elements of the last row of $A_{m}$ (denoted as $\left.a_{j}^{m}, j=1, \ldots, n\right)$, the control gain $b_{m}$ and the input signal $r(t)$ are assumed to be known by design.

Using a forward Euler's method with sampling period $T_{s} \in \mathbb{R}^{+}$ (Rugh, 1996) one obtains the following discretization for (1)-(3):

$x(k+1)=\tilde{A}(k) x(k)+\tilde{B} u(k)$,

$x_{m}(k+1)=\tilde{A}_{m} x_{m}(k)+\tilde{B}_{m} r(k)$,

with $\tilde{A}(k)=\left[\square_{n}+T_{s} A(k)\right], \tilde{B}=B \tilde{b}, \tilde{b}=T_{s} b, \tilde{A}_{m}=\left[\square_{n}+T_{s} A_{m}\right], \tilde{B}_{m}=B \tilde{b}_{m}$ $\tilde{b}_{m}=T_{s} b_{m} ; \mathbb{\square}_{n}$ being the $n \times n$ identity matrix. Note that $T_{s}$ is chosen so that $\tilde{A}_{m}$ is a discrete Hurwitz matrix.

The control objective is to find a feedback law of the form:

$u(k)=L_{X}^{\top}(k) x(k)+L_{R}(k) r(k)$,

where $L_{X}(k) \in \mathbb{R}^{n}$ and $L_{R}(k) \in \mathbb{R}$ are appropriately chosen adaptive gains, so as to ensure that the tracking error variable defined as $e(k)=x_{m}(k)-x(k)$

$\lim _{k \rightarrow \infty} e(k)=0$.

Given the control scheme sketched in Fig. 1, we have the following result.

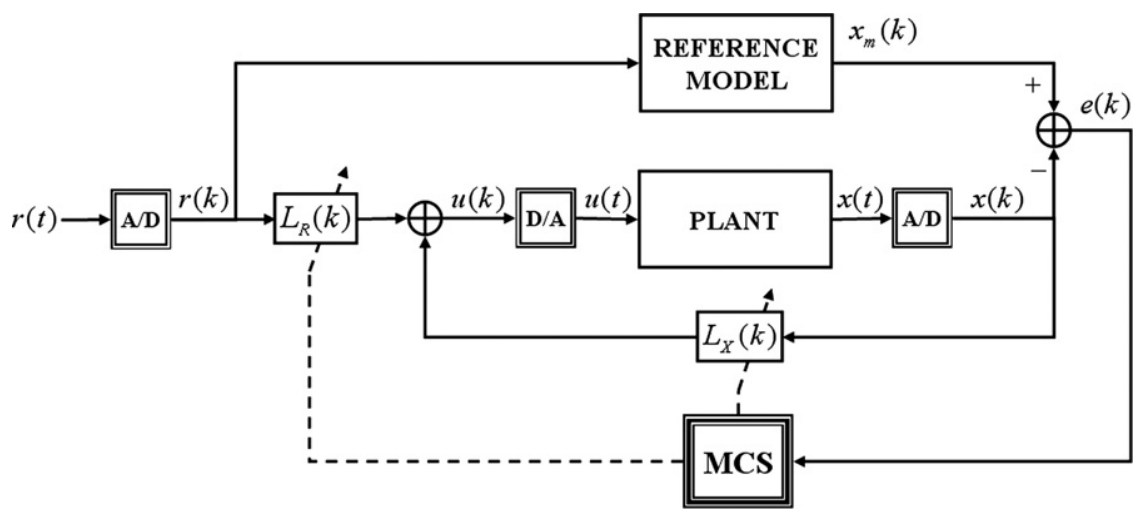

Fig. 1. Digital MCS scheme 
Theorem 1. Assume there exist symmetric, positive-definite matrices $P$ and $Q$ such that

$\tilde{A}_{m}^{\top} P \tilde{A}_{m}-P=-Q$

and let us set the system output as $y:=P e(k)$. Let us also assume that the $n$-th component of the output denoted by $y_{n}(t)$ is such that

$y_{n}(k+1) x_{j}(k)+\frac{a_{j}(k)-a_{j}(k-1)}{\alpha b} \approx y_{n}(k+1) x_{j}(k) \forall k \geq 1$.

Then, the adaptive control law (6) with gains selected as

$L_{X}^{\top}(k)=\alpha \sum_{i=0}^{k} y_{n}(i+1) x^{\top}(i)+\beta y_{n}(k+1) x^{\top}(k)$,

$L_{R}(k)=\alpha \sum_{i=0}^{k} y_{n}(i+1) r(i)+\beta y_{n}(k+1) r(k)$,

where $L_{X}(0)=0, \quad L_{R}(0)=0, \quad$ and $\alpha, \beta \in \mathbb{R}$ are such that $\operatorname{sign}(\alpha)=\operatorname{sign}(\beta)=\operatorname{sign}(b)$, guarantees that

$\lim _{k \rightarrow \infty} e(k)=0$.

Notice that both control gains, $L_{X}$ and $L_{R}$, have integral and proportional terms. It is worth remarking that the use of integral + proportional adaptation has a beneficial effect upon the convergence of the generalized state error vector in comparison to the use of integral adaptation, specially at the beginning of the adaptation process (Landau, 1979).

Proof. The proof relies on showing that the closed-loop error dynamics (11) and (12) can be rewritten as a feedback asymptotically hyperstable system (Popov, 1973; Landau, 1979), thus guaranteeing asymptotic convergence of the tracking error. It can be derived according to the following steps:

- Step 1: Recast the error dynamics as a feedback system.

- Step 2: Show that the feedback block satisfies a Popov inequality.

- Step 3: Prove that the feedforward path is strictly positive real.

\subsection{Step 1}

Considering the system output to be $y(k)=C e(k)$ with $C \equiv P$, the tracking error dynamics (1)-(3) can be written as

$e(k+1)=\tilde{A}_{m} e(k)+\square_{n} w(k+1)$

$y(k)=C e(k)$

where

$w(k+1)=\left[\tilde{A}_{m}-\tilde{A}(k)-\tilde{B} L_{X}^{\top}(k)\right] x(k)+\left[\tilde{B}_{m}-\tilde{B} L_{R}(k)\right] r(k)$.

System (11-12) is the negative feedback interconnection of an LTI block with dynamic matrix $\tilde{A}_{m}$, input matrix $\mathbb{\square}_{n}$ and output matrix $C$, while the feedback path is a nonlinear

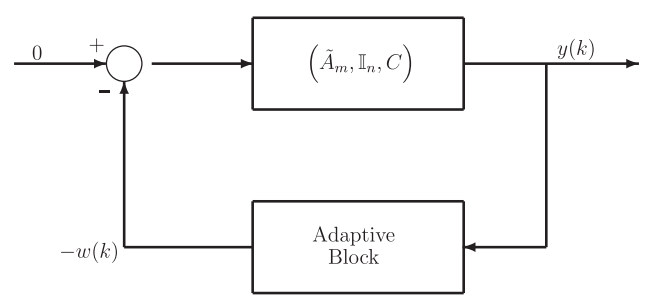

Fig. 2. Closed-loop error dynamics represented as an equivalent feedback system. time-varying block that maps $y(k)$ onto $-w(k)$ (see Fig. 2 for a schematization of the tracking error system).

It is worth pointing out here that the control action (6) can be rewritten as

$u(k)=L^{\top}(k) v(k)$

where

$v^{\top}(k):=\left[x^{\top}(k) r(k)\right] \in \mathbb{R}^{n+1}$

and

$L^{\top}(k):=\left[L_{X}^{\top}(k) L_{R}(k)\right] \in \mathbb{R}^{n+1}$.

Then, recalling (13), $w(k+1)$ becomes

$w(k+1)=\Phi(k) v(k)$

with

$\Phi(k)=\left[\tilde{A}_{m}-\tilde{A}(k)-\tilde{B} L_{X}^{\top}(k) \tilde{B}_{m}-\tilde{B} L_{R}(k)\right] \in M_{n \times(n+1)}(\mathbb{R})$.

It is to see that $\tilde{B} L_{X}^{\top}(k)$ is a matrix with null elements everywhere except in the last row. Then, the adaptive control gains in $L_{X}$ will not be able to cope with the mismatch between the discretized reference model and plant matrices unless $\tilde{A}_{m}-\tilde{A}(k)$ shares this specific structure. This is, indeed, the cornerstone of the procedure: the requirement of having these single matrices in control canonical form is not a necessary but a sufficient condition.

A forward Euler discretization makes $\tilde{A}_{m}, \tilde{A}(k)$ loose the control canonical form initially assumed for the corresponding continuous-time matrices $A_{m}, A(t)$. However, it allows $\Phi(k)$ to be rewritten as

$\Phi(k)=T_{s}\left[A_{m}-A(k)-B b L_{X}^{\top}(k) B\left(b_{m}-b L_{R}(k)\right)\right] \in M_{n \times(n+1)}(\mathbb{R})$.

Step 2

The Popov inequality for the feedback block reads as

$\sum_{k=0}^{l} y(k+1)^{\top}[-w(k+1)] \geq-\gamma^{2}, \quad \gamma \in \mathbb{R}, \quad \forall l \geq 0$.

The proof is purely algebraic and is presented in Appendix A.

Step 3

According with the theorem assumption, $\tilde{A}_{m}$ is a discrete Hurwitz matrix and, thus, the discrete reference model (5) is asymptotically stable. Hence, the feedforward path, composed by the linear time-invariant block characterized by the transfer function:

$H(z)=C z\left(z \rrbracket_{n}-\tilde{A}_{m}\right)^{-1}$

is strictly positive real, because matrix $C$ has been selected following the discrete positive real lemma (Landau, 1979, Theorem C-4, pp. 386).

Therefore, the theorem remains proved.

Remark 1. Notice that, defining

$L_{I j}(k)=\alpha \sum_{i=0}^{k} y_{n}(i+1) x_{j}(i), \quad j=1, \ldots, n$,

assumption (8) may be equivalently rewritten as

$L_{I j}(k)-L_{I j}(k-1)+\frac{a_{j}(k)-a_{j}(k-1)}{b} \approx L_{I j}(k)-L_{I j}(k-1) \quad \forall k \geq 1$.

Hence, (8) indicates that the plant parameters must vary less rapidly than the discrete-time integral part of the adaptive control gains. 


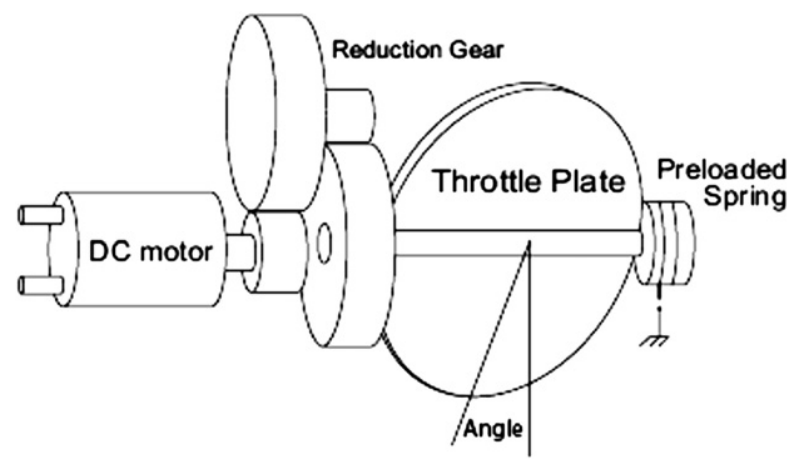

Fig. 3. Schematic diagram of an Electronic Throttle Body (ETB).

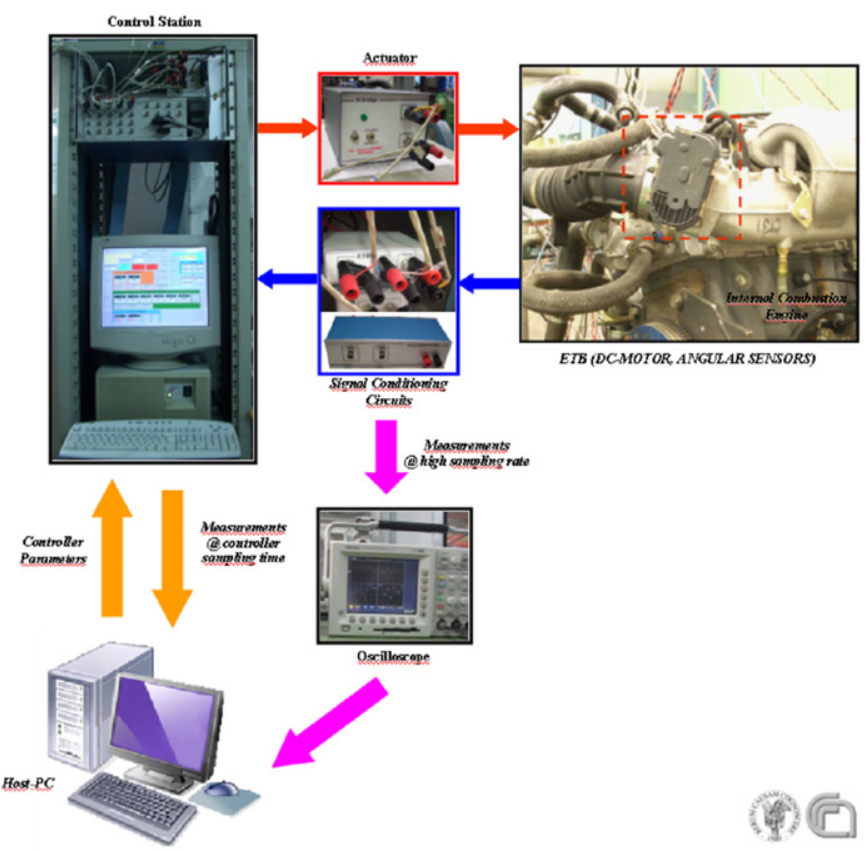

Fig. 4. Schematization of the experimental setup.

The slow variation of the system parameters with respect to the adaptive gains is a technical assumption typical of the MCS approach, both in continuous-time and discrete-time but, so far, no results are available to test if this is verified a priori. When this hypothesis is not verified $e(k) \rightarrow 0$ can be no longer guaranteed. Instead, following Landau (1979) and recasting the plant matrix as

$A(k)=A_{N}+\Delta A(t)$,

one may leave $A_{N}$ in the feedback path of Fig. 2 and consider the remaining term, $T_{s} \Delta A(k) x(k)$, as an input signal to the system. In turn, the feedforward and feedback paths are strictly positive real and satisfy the Popov inequality, respectively. Hence, the closed-loop error system will be finite-gain $L_{2}$ stable.

\section{The electronic throttle body}

In modern combustion engines the driver input (through the accelerator) is elaborated, together with other requirements, into a torque demand. The effective torque is achieved by managing the air mass flow rate (di Gaeta, Montanaro, \& Giglio, 2011, 2010).
The ETB is the mechatronic device used to regulate the air flow coming into the internal combustion engine. It is located between the air filter box and the intake manifold. When the throttle plate opens an airflow sensor detects this change and communicates it to the ECU. The control signal generated by the ECU is transduced, by means of an $\mathrm{H}$-bridge power converter, into the armature voltage of a dc-motor. The rotation motion is then transferred from the motor shaft to the plate shaft through a gear system. A schematic of the ETB is shown in Fig. 3. Despite its apparent simplicity, the system behavior is affected by many nonlinearities which can dramatically alter its dynamics such as a piecewise linear restoring torque, friction, impacts and backlash (see di Bernardo, di Gaeta, Montanaro, et al., 2010 for further details). Moreover, the system parameters are often uncertain because of unavoidable manufacturing tolerances, variable operating conditions and mechanical wear (Rossi, Tilli, \& Tonielli, 2000).

With the alternative aim of industrializing the device, different control approaches have been proposed in the technical automotive literature. Often classical controllers, for example those based on a PID structure are used (Pavkovic, Deur, Jansz, \& Peric, 2003; Deur, Pavkovic, Peric, Janz, \& Hrovat, 2004; Pavković, Deur, Jansz, \& Perić, 2006), but they are equipped with some feedforward model-based action to compensate the nonlinearities acting on the ETB, mainly due to the presence of friction (see, for example, de Witt, Olsson, Astrom, \& Lischinsky, 1995, 2001; Dagci, Pan, \& Ozguner, 2002). Further control techniques are based on constrained optimal control (Vasak, Baoti, Morari, Petrovic, \& Peric, 2006), robust methods (Rossi et al., 2000) and hybrid approaches (Vasak, Baoti, Petrovic, \& Peric, 2007, 2004; Yokoyama \& Shimizu, 1998; Dagci et al., 2002; Pan, Dagci, \& Ozguner, 2001; Ozguner, Hong, \& Pan, 2001), but again they are based on a good knowledge of the plant dynamics. Some further approaches do not require a priori knowledge of the plant nonlinearities, e.g. those presented in Pavkovic et al. (2003), Deur et al. (2004), and Pavković et al. (2006).

For its features and relevance, the ETB has been often chosen as an ideal case study to investigate the performance and robustness of adaptive control schemes in the presence of model uncertainties and disturbances as discussed, for example, in di Bernardo, di Gaeta, Montanaro, et al. (2010), Alt, Blath, Svaricek, and Schultalbers (2010), Barić, Petrović, and Perić (2005), Pavković et al. (2006), and Corno, Tanelli, Savaresi, and Fabbri (2011).

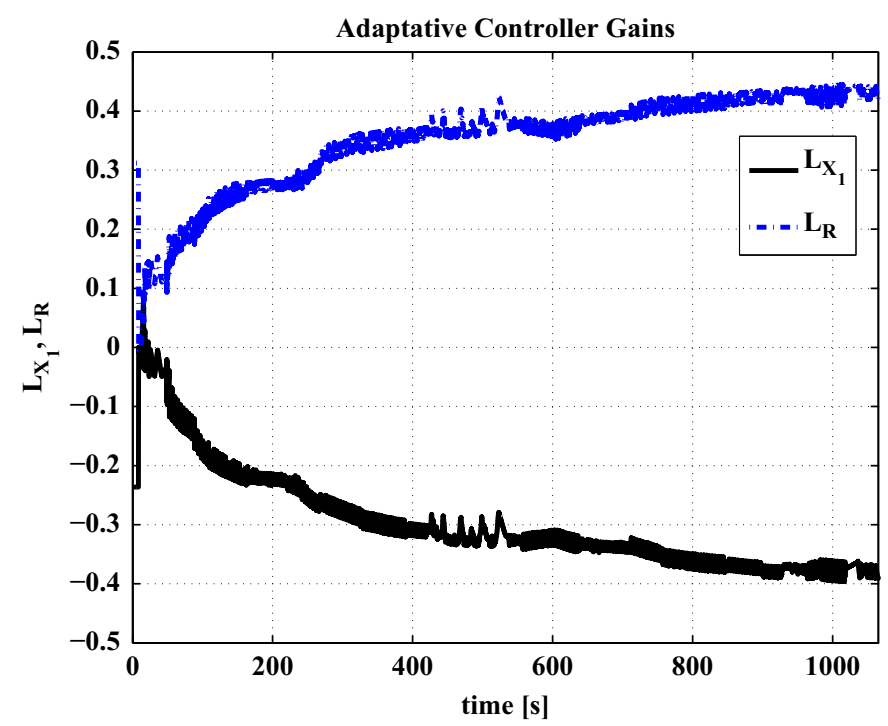

Fig. 5. Evolution of the adaptive gains: $L_{X_{1}}$ (solid black) and $L_{R}$ (dashed blue). (For interpretation of the references to color in this figure legend, the reader is referred to the web version of this article.) 
Therefore, ETB is an excellent device to test the adaptive law discussed in Section 2 relying on a minimal knowledge of the plant. For this reason an accurate mathematical model of the plant is not strictly necessary for control synthesis and its digital implementation. Nevertheless, for the sake of clarity and to emphasize the highly nonlinear nature of the plant and its open-loop dynamics, its mathematical model and some evidence of its dynamical behavior (see also di Bernardo, di Gaeta,
Montanaro, et al., 2010; di Bernardo et al., 2009) are presented in Appendix B.

\section{Discrete-time MCS control of the ETB}

As only minimal knowledge of the plant is required for the control synthesis, the first step for the control design is the choice
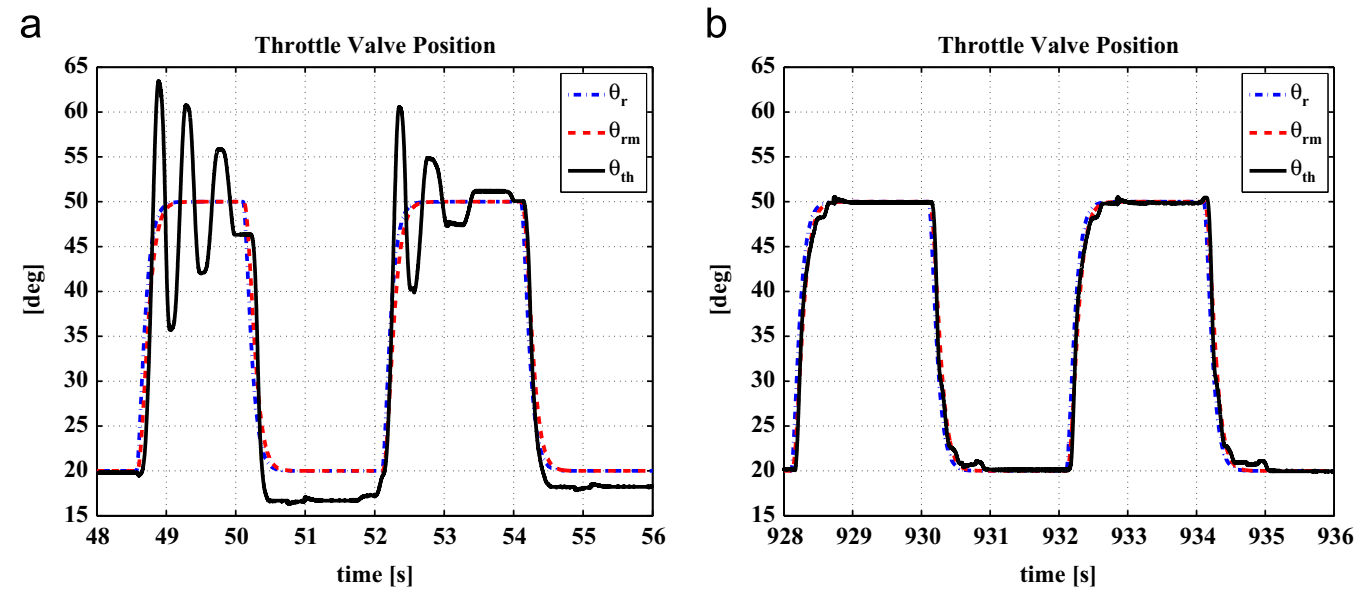

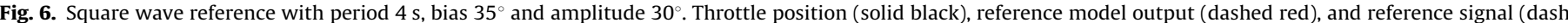

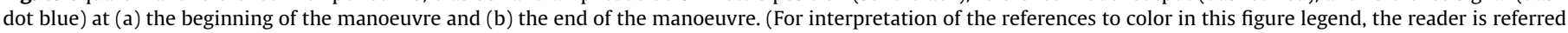
to the web version of this article.)
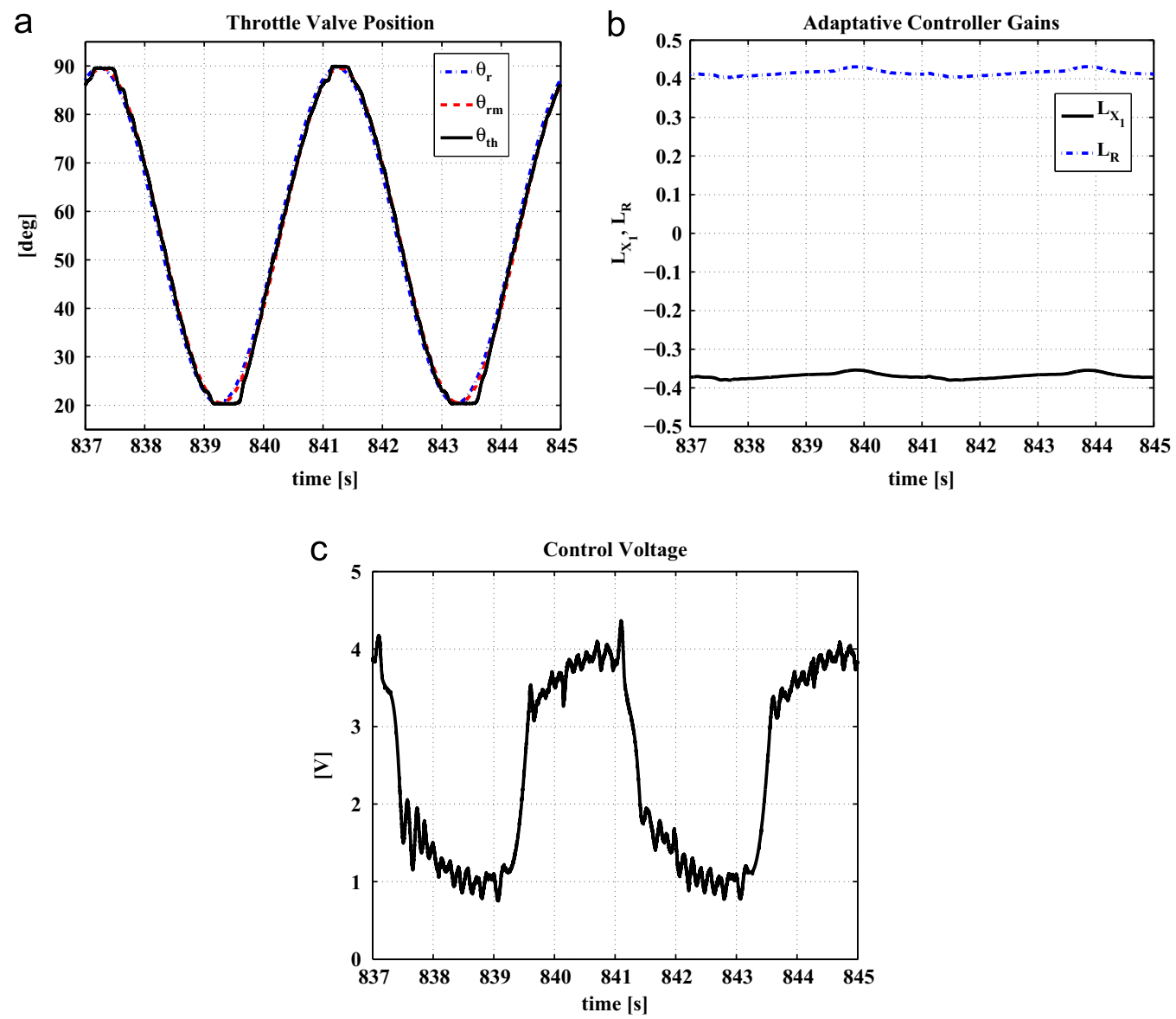

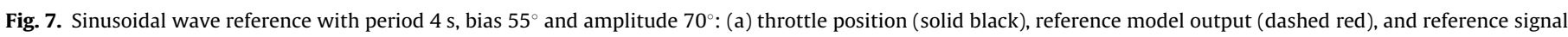

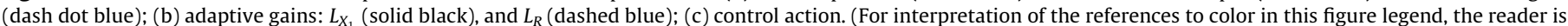
referred to the web version of this article.) 
of an LTI reference model. Here, a second-order LTI model in control canonical form is selected. The parameters of the reference model are chosen so as to guarantee a settling time of about $135 \mathrm{~ms}$, no oscillatory behavior and unitary gain. Recall that the settling time is defined as the minimum time after which the throttle plate angle remains within $5 \%$ of its steady-state value.

This continuous-time model has been discretized via a forward Euler's method in order to derive the discretized version to be used in the control loop. As common in MCS applications (see, for example, Stoten \& Gómez, 2001), the adaptive weights $\alpha$ and $\beta$ have been selected heuristically as a trade-off between convergence time and reactivity of the control action so that $\alpha / \beta=10$. Here we choose $\alpha=50 \times 10^{-9}$ and $\beta=5 \times 10^{-9}$. These values were found to produce a realistic amplitude of the control input during the experimental validation.

According to the control law (6), (9), (10), the computation of the control gains at the current time instant $k$ requires knowledge of the plant output at the next time instant, namely $y_{n}(k+1)$. As remarked in the classical work of Landau (1979), this problem can be solved by means of an estimate of $y_{n}(k+1)$, say $\hat{y}_{n}(k+1)$, that can be calculated using the values of the system variables available up to the instant $k$. Namely,

$y_{n}(k+1)=\frac{\hat{y}_{n}(k+1)}{1+T_{s} c_{n n} b(\alpha+\beta)\left[x(k)^{\top} x(k)+r^{2}(k)\right]}$.

However, as shown in Landau (1979), the accuracy of the estimation $\hat{y}_{n}(k+1)$ relies on knowledge of $A(k)$. This is in contradiction with the MCS philosophy, which is based on minimal knowledge of the system dynamics. The practical solution adopted in this work is to follow the approach of Landau (1979) and replace $\hat{y}_{n}(k+1)$ in (21) by $y_{n}(k)$, which is claimed to be a good prediction if the sampling period is short (Landau, 1979). Experimental results reported in Section 4.2 will show that, despite its simplicity, this choice yields a successful implementation of the control strategy. Concerning the estimation of parameter $b$, and again in accordance with Landau (1979), $b$ should be replaced by any value that falls within its range of variation, which is assumed to be known. In the present case $b$ has been directly identified from the experimental step response of the system.

A Smooth Trajectory Reference (STR) first-order filter is introduced to limit the tracking error during tip-in/tip-out conditions. This is a usual experimental tool to emulate the realistic case when the throttle is in the gas pedal following mode, whereby the reference may be burdened with noise and perturbations due to mechanical vibrations (see Rossi et al., 2000, for example, for further details). The sample time is chosen as $T_{s}=1 \mathrm{~ms}$ in agreement with common automotive hardware limitations and leads to a stable $\tilde{A}_{m}$.

Note that, as the velocity of the plate is not available, a proper derivative filter has to be used to reconstruct the plate velocity. In so doing, unavoidable noise and delays are introduced in the closed-loop system along the velocity channel affecting the overall control performance. A way to overcome this problem in the automotive literature (e.g., Vasak \& Petrovic, 2003) is the use of state observers to estimate the velocity. Despite being a viable approach, the use of observers goes against the typical industrial requirements of reducing as much as possible the processing time and memory usage needed to implement controllers in an on-board vehicle ECU. Therefore, here we took an unorthodox a

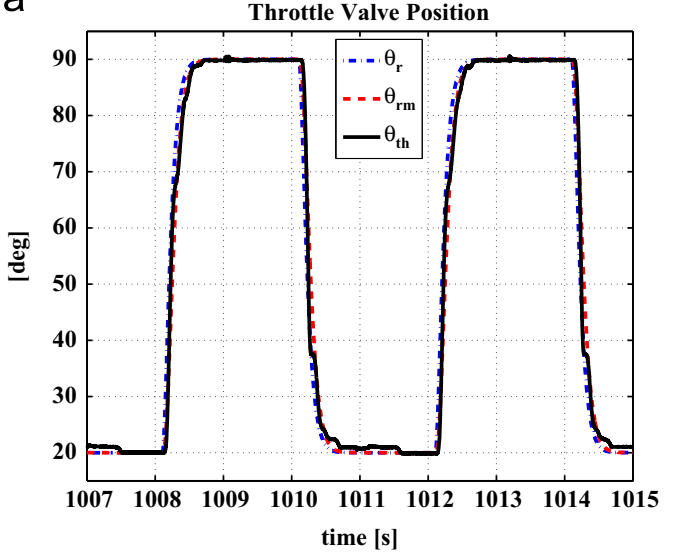

b

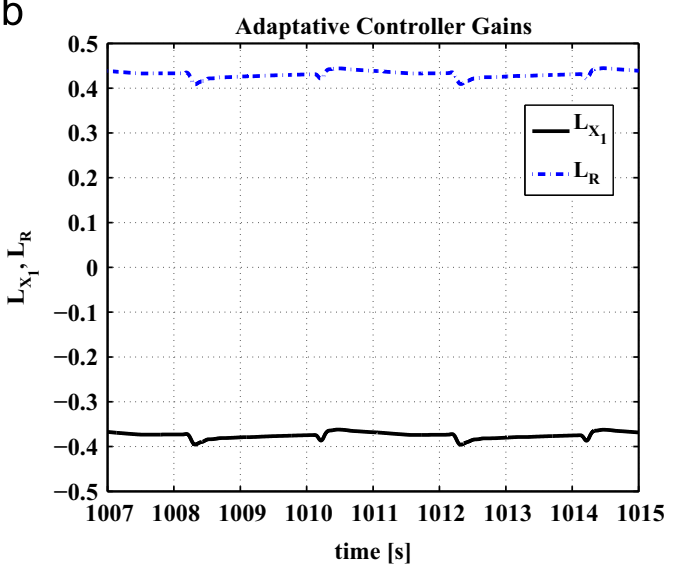

C

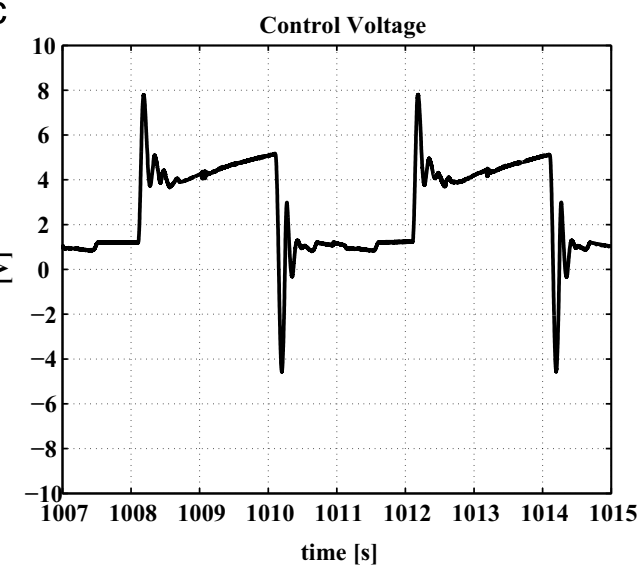

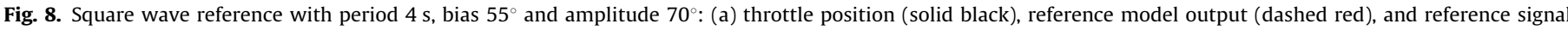

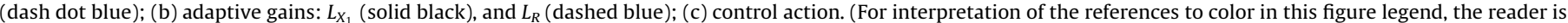
referred to the web version of this article.) 
implementation choice justified by the previous observations that the MCS algorithms performs well in a number of non-canonical situations (see for example di Bernardo, di Gaeta, Montanaro, et al., 2010). In practice, the algorithm designed taking into account a full state feedback was implemented by using only measurements of the angular position. Indeed, this is empirical and unusual choice can, on the other hand, also offer extra evidence of the robustness and ease of implementation of this novel control approach.

\subsection{Experimental setup}

The experimental setup consists of (i) an Electronic Throttle Body (DV-E5, Bosch), embedding a DC motor; (ii) two dual resistive angular position sensors; (iii) a battery voltage sensor; (iv) an H-bridge power circuit (to drive the DC motor); (v) a Hall effect current sensor (LTA 50P/SP1, LEM); (vi) signal conditioning circuits; (vii) a station for Rapid Control Prototyping (RCP) (see also Fig. 4 for its schematization).

The open-loop response of the ETB can be summarized through two characteristic times. Namely, the time necessary to fully open the valve under a battery voltage step $\left(V_{b a t} \approx 12[\mathrm{~V}]\right)$, $t_{\text {open }} \approx 100 \mathrm{~ms}$, and the current-less return time in open loop condition, namely the time necessary to close the valve in free evolution, $t_{c l} \approx 350 \mathrm{~ms}$.

The RCP is a dSpace based Multiprocessor System equipped with the DS1003 (DSP TMS320C40, 60 Mflops) and DS1004 (DEC Alpha AXP 21164, 600 Mflops) processor boards. An analog DS2201 (20 ch., 12 bit, $30 \mathrm{kHz}$ ) and a digital DS4002 (8 ch. CAP/
CMP res. $30 \mathrm{bit} / 200 \mathrm{~ns}$, freq. max. $833 \mathrm{kHz}$ ) board allow the I/O handling. The DSP is programmed in Matlab/Simulink (MathWorks) environment and the experiments are managed and instrumented by a ControlDesk application (dSpace). Furthermore, an oscilloscope (TDS-3014, Tektronix) is used to perform high frequency measurements.

Note that, using the hardware described above, the control tasks are discretized with a sampling time of $1 \mathrm{~ms}$. Furthermore the turnaround time, i.e. the duration of the control task, is $10 \mu \mathrm{s}$ when the MCS adaptive algorithm is used to steer the ETB dynamics.

\subsection{Experimental results}

The proposed controller has been exhaustively tested over a long reference signal of the throttle position composed by a mixed sequence of canonical signals, including square, sinusoidal, and step functions as well as free driver commands.

One can clearly see that, as time increases, the control gains evolve as shown in Fig. 5 yielding a better and better tracking of the reference model state. The effects of the gain adaptation on the tracking error are shown in Fig. 6, where the reference position and the valve position are plotted close to the beginning of the test and after about $850 \mathrm{~s}$. Specifically, the overshoot, undershoot and the oscillations present at the beginning of the manoeuvre (Fig. 6a) disappear as the controller gains adapt as shown in Fig. 6b.

The closed-loop behavior of the ETB during a set of manoeuvres where the valve is required to open and close over a
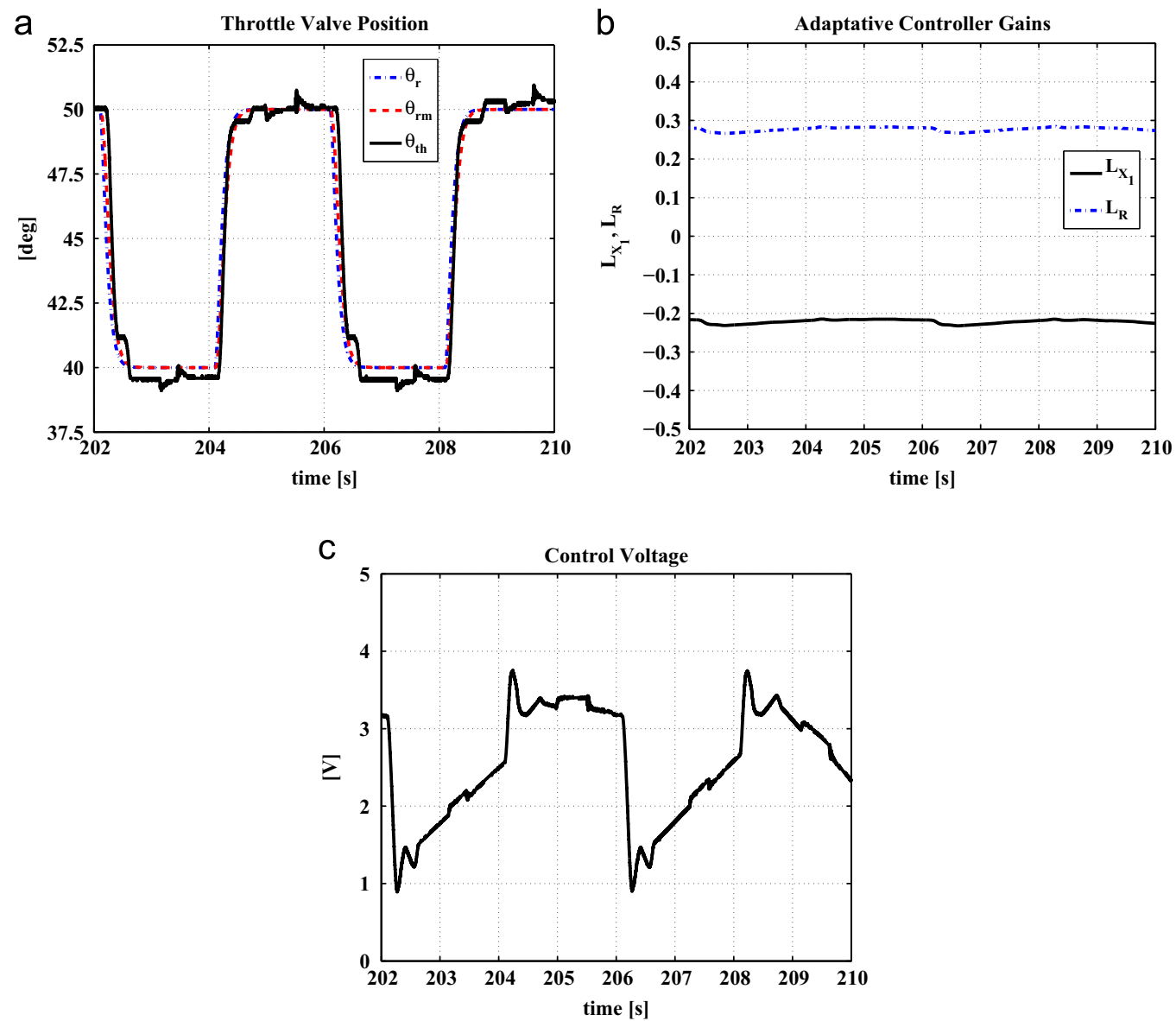

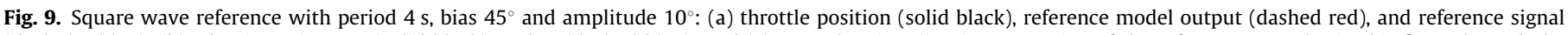

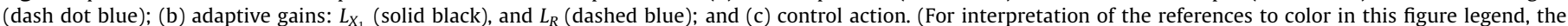
reader is referred to the web version of this article.) 
position range of about $70^{\circ}$ is shown in Fig. 7 . This is a particularly challenging problem as the restoring force is, in practice, characterized by different elastic coefficients depending on the actual position of the plate. Despite the lack of any explicit modeling of such spring behavior, experiments confirm that the adaptive controller does indeed guarantee good tracking performance in these operating conditions. A slight loss of performance can be observed in the reversal phase, which is characterized by large amounts of friction. This is due to the fact that, as previously observed, the controller is not using velocity measurements. Hence, performance could be increased by adding a velocity observer or, as usual in the automotive literature, by adding further actions devoted to the direct compensation of the effects of friction in specific working conditions. Notice that, in turn, this control approach does not require a direct compensation of the many nonlinearities acting on the plant.

Good responses are again observed when a square wave reference signal is used, as shown in Fig. 8. In both cases, the corresponding control input (Figs. 7(b) and 8(b)) and gain evolution (Figs. 7(c) and 8(c)) remain within the admissible range.

Another significant test is the case of small amplitude reference signals, when the presence of stick-slip motion due to friction becomes particularly relevant because position and velocity variations are relatively low. As shown in Fig. 9 the discretetime MCS controller copes well with such unwanted nonlinear perturbations, though small steady state errors arise due to the already commented lack of velocity measurements. However, recall that these disturbances are not taken explicitly into account during the control design. Similar results were obtained for different amplitude signals and are not reported here for the sake of brevity.

Further validation of the controller was carried out on more realistic free driver commands, as shown in Fig. 10. Also in this practical case the tracking is excellent while the control action is again within admissible bounds. It is worth mentioning here that performing adaptation tests over a long manoeuvre can be useful to implement an automatic procedure for the tuning of the controller gains before the production/assembling phase. Namely, the gains could be set to the final steady-state values obtained at the end of the manoeuvre and then adapted about those values to cope with uncertainties and external disturbances (see Fig. 5).

Finally, to better illustrate the advantages of performing the direct synthesis of the MCS algorithm in discrete time, further experimental results are presented which were obtained implementing the classical time continuous MCS algorithm (see Stoten \& Benchoubane, 1990a) discretized via Tustin method. The reference signal is obtained concatenating three signals, namely, a square wave (period $4 \mathrm{~s}$, bias $30^{\circ}$ and amplitude $20^{\circ}$ ), a sinusoidal wave (period $4 \mathrm{~s}$, bias $55^{\circ}$ and amplitude $70^{\circ}$ ) and then again the previous square wave. The reference model and sampling time are the same as in the discrete time algorithm. The adaptation weights are set empirically to $\alpha=0.5$ and $\beta=0.05$. Although excellent tracking performance is observed in simulation (see Appendix B for a description of the ETB model), once the strategy is implemented for its experimental validation on the device, the unavoidable presence of noise and hidden dynamics due to the discretization of the adaptation law drastically jeopardize the tracking performance (see Fig. 11a). Specifically, at the first change of the reference a

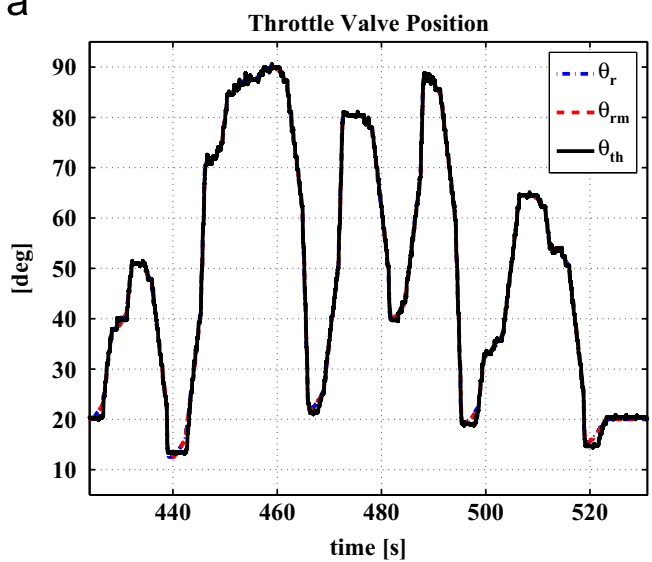

b

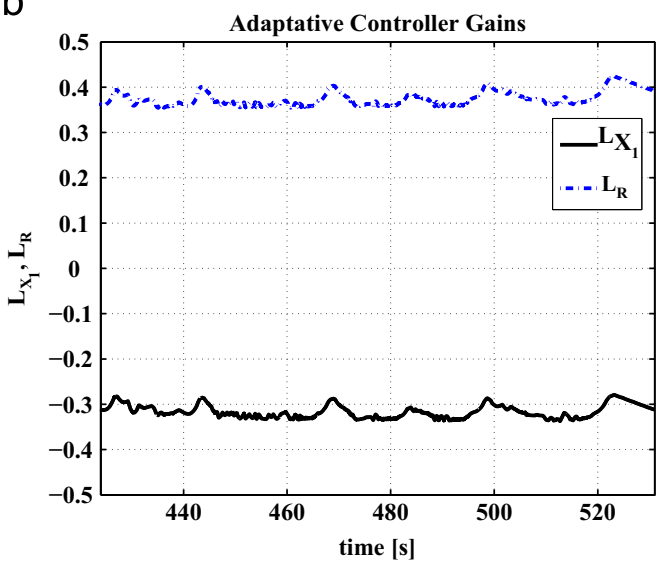

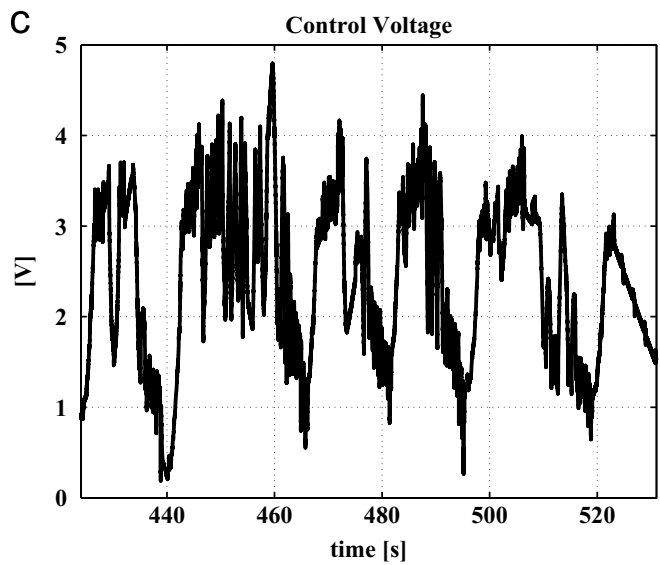

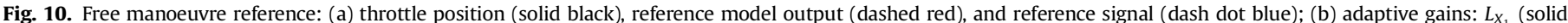

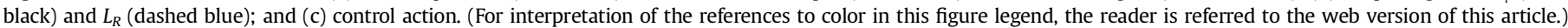


a

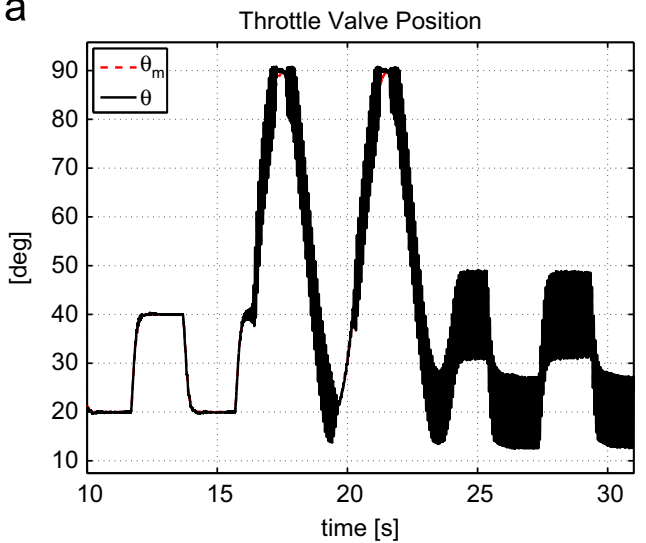

b

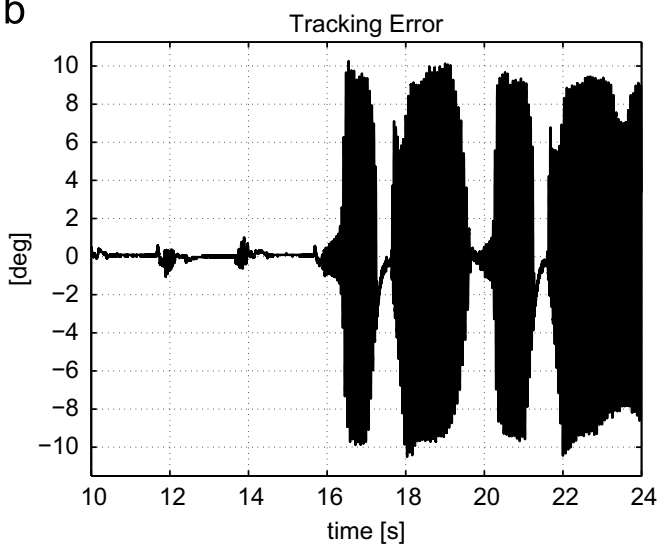

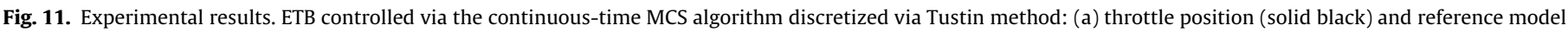
output (dashed red) and (b) tracking error. (For interpretation of the references to color in this figure legend, the reader is referred to the web version of this article.)

input signal, unwanted persistent oscillations around the reference trajectory are induced. As shown in Fig. $11 \mathrm{~b}$, the tracking error oscillates (between $-10^{\circ}$ and $10^{\circ}$ ) independently from the demanded model reference dynamics. This is in accordance with the significant reduction of the stability regions and the loss of dynamic performance theoretically predicted in Bursi et al. (2009) when discretizing the continuous-time MCS algorithm.

\section{Conclusions}

This paper discussed the design, experimental implementation and proof of stability of a discrete-time MCS control algorithm originally presented in di Bernardo, di Gennaro, Olm et al. (2010) showing that it represents an effective and viable option to control discretized dynamical systems. In particular, the proposed adaptive control strategy was able to cope with the dynamics of a highly uncertain and nonlinear plant such as the ETB device. An extension of this discrete-time MRAC algorithm for improving tracking performance and decreasing the settling time of the adaptive gains is currently under investigation and will be presented elsewhere.

\section{Acknowledgments}

J.M. Olm was partially supported by the Spanish Ministerio de Educación (MEC) under project DPI2010-15110.

\section{Appendix A. Step 2 of the proof of Theorem 1}

Taking into account (9), (10), (15) and (16), one can decompose the control gain vector $L(k)$ as the sum of an integral term, $L_{I}(k)$, and a proportional term, $L_{P}(k)$ :

$L^{\top}(k)=L_{I}(k)+L_{P}(k), \quad L_{I}(k), L_{P}(k) \in \mathbb{R}^{n+1}$

with

$L_{I j}(k)=\alpha \sum_{i=0}^{k} y_{n}(i+1) v_{j}(i), \quad L_{P j}(k)=\beta y_{n}(k+1) v_{j}(k)$.

Then, given the assumption of canonical form for continuoustime systems (1) and (3), one gets from (18) that

$\Phi(k)=T_{S} B\left[\phi_{I}(k)+\phi_{P}(k)\right]^{\top}$,

where $\phi_{I}(k), \phi_{P}(k) \in \mathbb{R}^{n+1}$ are such that

$\phi_{I j}(k)=a_{j}^{m}-a_{j}(k)-b L_{I j}(k), \quad j=1, \ldots, n$,

$$
\begin{aligned}
& \phi_{I n+1}(k)=b_{m}-b L_{I n+1}(k), \\
& \phi_{P j}=-b L_{P j}(k), \quad j=1, \ldots, n+1 .
\end{aligned}
$$

Lemma 1. The expression in the left hand side of (19) may be written as

$\sum_{k=0}^{l} y(k+1)^{\top}[-w(k+1)]=\sum_{j=1}^{n+1} S_{I j}+\sum_{j=1}^{n+1} S_{P j}$

with

$S_{I j}=T_{S} \sum_{k=0}^{l} y_{n}(k+1)\left[-\phi_{I j}(k)\right] v_{j}(k)$,

$S_{P j}=T_{S} \sum_{k=0}^{l} y_{n}(k+1)\left[-\phi_{P j}(k)\right] v_{j}(k)$.

Proof. Notice that, recalling (17),

$\sum_{k=0}^{l} y(k+1)^{\top}[-w(k+1)]=\sum_{k=0}^{l} y^{\top}(k+1)[-\Phi(k)] v(k)$

and, therefore,

$\sum_{k=0}^{l} y^{\top}(k+1)[-\Phi(k)] v(k)=T_{S} \sum_{k=0}^{l} y^{\top}(k+1) B\left[-\phi_{I}(k)-\phi_{P}(k)\right]^{\top} v(k)$

$$
\begin{aligned}
& \sum_{k=0}^{l} y^{\top}(k+1)[-\Phi(k)] v(k)=T_{S} \sum_{k=0}^{l} y_{n}(k+1)\left[-\phi_{I}(k)\right]^{\top} v(k) \\
& \quad+T_{S} \sum_{k=0}^{l} y_{n}(k+1)\left[-\phi_{P}(k)\right]^{\top} v(k)=S_{I}+S_{P} .
\end{aligned}
$$

Now

$S_{I}+S_{P}=\sum_{j=1}^{n+1} S_{I j}+\sum_{j=1}^{n+1} S_{P j}$,

with $S_{I j}, S_{P j}$ defined as in (A.5) and (A.6), respectively, and the result follows.

Then, it is sufficient to prove that

$S_{I j} \geq-\gamma_{j}^{2}, \quad \gamma_{j} \in \mathbb{R}, \quad S_{P j} \geq 0 \quad \forall j=1, \ldots, n+1 \quad \forall l \geq 0$.

With respect to the first inequality, $S_{I j} \geq-\gamma_{j}^{2}$, notice that one has to take into account expression (A.2) for $j=1, \ldots, n$, while for 
$j=n+1$ equality (A.3) holds. Let us then define, for $j=1, \ldots, n$,

$z_{j}(k)=\sum_{i=0}^{k} y_{n}(i+1) v_{j}(i)+\frac{a_{j}(k)-a_{j}^{m}}{\alpha b}$,

$z_{j}(-1)=\frac{a_{j}(0)-a_{j}^{m}}{\alpha b}$.

Lemma 2. For $j=1, \ldots, n$, the assumption that the system parameters $a_{j}(k)$ satisfy (8) yields

$S_{I j}=\alpha b T_{S} \sum_{k=0}^{l} z_{j}(k)\left[z_{j}(k)-z_{j}(k-1)\right]=\alpha b T_{S}\left[\sum_{k=0}^{l} z_{j}^{2}(k)-\sum_{k=0}^{l} z_{j}(k) z_{j}(k-1)\right]$.

Proof. On the one hand, when $j=1, \ldots, n$, it follows from (A.5) and (A.2) that

$$
\begin{aligned}
S_{I j} & =T_{S} \sum_{k=0}^{l} y_{n}(k+1) v_{j}(k)\left(b L_{I j}(k)+a_{j}(k)-a_{j}^{m}\right) \\
& =T_{S} \alpha b \sum_{k=0}^{l} y_{n}(k+1) v_{j}(k)\left(\sum_{i=0}^{k} y_{n}(i+1) v_{j}(i)+\frac{a_{j}(k)-a_{j}^{m}}{\alpha b}\right) .
\end{aligned}
$$

On the other hand, the assumption that the system parameters $a_{j}(k)$ satisfy (8) yields

$z_{j}(k)-z_{j}(k-1)=y_{n}(k+1) v_{j}(k)+\frac{a_{j}(k)-a_{j}(k-1)}{\alpha b} \approx y_{n}(k+1) v_{j}(k)$,

because $v_{j}(k)=x_{j}(k)$, for all $j=1, \ldots, n$ (see (15)).

Then, it is immediate that

$S_{l j}=\alpha b T_{s} \sum_{k=0}^{l} z_{j}(k)\left[z_{j}(k)-z_{j}(k-1)\right]=\alpha b T_{s}\left[\sum_{k=0}^{l} z_{j}^{2}(k)-\sum_{k=0}^{l} z_{j}(k) z_{j}(k-1)\right]$.

Lemma 3. One has that

$\sum_{k=0}^{l} z_{j}(k) z_{j}(k-1) \leq\left|z_{j}(0) z_{j}(-1)\right|+\sum_{k=0}^{l} z_{j}^{2}(k)$.

\section{Proof. Set}

$q_{1}^{\top}=\left[z_{j}(0), z_{j}(1), \ldots, z_{j}(l)\right], \quad q_{2}^{\top}=\left[z_{j}(1), \ldots, z_{j}(l), 0\right]$.

Then, using the Cauchy-Schwarz inequality, it follows for all $l \geq 0$ that:

$$
\begin{aligned}
\sum_{k=0}^{l} z_{j}(k) z_{j}(k-1) & \leq\left|\sum_{k=0}^{l} z_{j}(k) z_{j}(k-1)\right| \leq\left|z_{j}(0) z_{j}(-1)\right|+\left|q_{1}^{\top} q_{2}\right| \\
& \leq\left|z_{j}(0) z_{j}(-1)\right|+\left\|q_{1}\right\|\left\|q_{2}\right\| \leq\left|z_{j}(0) z_{j}(-1)\right|+\left\|q_{1}\right\|^{2} \\
& =\left|z_{j}(0) z_{j}(-1)\right|+\sum_{k=0}^{l} z_{j}^{2}(k) .
\end{aligned}
$$

Hence, since $\operatorname{sign}(\alpha)=\operatorname{sign}(b)$ by assumption, it is immediate from Lemmas 2 and 3 that the generic term $S_{I j}$ can be made greater than a finite negative constant:

$S_{I j} \geq-\left|z_{j}(0) z_{j}(-1)\right| \quad j=1, \ldots, n$

For $j=n+1$ an equivalent procedure that takes into account expression (A.3) yields:

$$
\begin{aligned}
S_{I n+1} & =\alpha b T_{S} \sum_{k=0}^{l} y_{n}(k+1) v_{n+1}(k)\left(\sum_{i=0}^{k} y_{n}(i+1) v_{n+1}(i)-\frac{b_{m}}{\alpha b}\right) \\
& \geq-\gamma_{n+1}^{2} \quad \forall l \geq 0 .
\end{aligned}
$$

Regarding $S_{P j}$ notice that, according to (A.1) and (A.4), under the assumption that $\operatorname{sign}(\beta)=\operatorname{sign}(b)$, for all $j=1, \ldots, n+1$, it can be deduced that

$$
S_{P j}=T_{S} \sum_{k=0}^{l} y_{n}(k+1) b L_{P j} v_{j}(k)=\beta b T_{s} \sum_{k=0}^{l}\left[y_{n}(k+1) v_{j}(k)\right]^{2} \geq 0 \quad \forall l \geq 0 .
$$

\section{Appendix B. Detailed continuous-time mathematical model and experimental evidence of the open-loop nonlinear dynamics of the ETB}

A detailed mathematical model of the plant was derived in di Bernardo, di Gaeta, Montanaro, et al. (2010). The overall model is

$$
\left\{\begin{array}{l}
\frac{d i}{d t}=-\frac{R}{L} i-\frac{K_{v}}{L} \frac{G_{r}}{K} \omega_{t h}+\frac{1}{L} v_{a}, \\
\frac{d \theta_{t h}}{d t}=\omega_{t h}, \\
\frac{d \omega_{t h}}{d t}=\frac{K K_{t}}{J} G_{r} i-\frac{K}{J} T_{s}\left(\theta_{t h}\right)-\frac{K}{J} T_{f}\left(\omega_{t h}\right),
\end{array}\right.
$$

where $\theta_{\text {th }} \in\left[\theta_{\min }, \theta_{\max }\right][\mathrm{deg}]$ is the plate position, with $\theta_{\min }$ and $\theta_{\max }$ being the minimum and the maximum allowed angles, respectively; $\omega_{t h}[\mathrm{deg} / \mathrm{s}]$ is the velocity of the plate; $i[\mathrm{~A}]$ is the current through the coil of the armature; $v_{a} \in\left[-V_{\text {batt }}, V_{\text {batt }}\right][\mathrm{V}]$ is the voltage source across the coil of the armature ( $V_{\text {batt }}$ being the battery voltage); $L[\mathrm{H}]$ is the equivalent inductance of the armature coil; $R[\Omega]$ is the equivalent resistance of the armature coil; $K_{v}$ [V s/rad] is the velocity constant determined by the flux of the permanent magnets; $K_{t}[\mathrm{~N} \mathrm{~m} / \mathrm{A}]$ is the torque constant; $K=180 / \pi ; J\left[\mathrm{~kg} \mathrm{~m}^{2}\right]$ is the equivalent moment of inertia; $G_{r}$ is the transmission ratio due to the gear; $T_{s}[\mathrm{~N} \mathrm{~m}]$ is the torque due to the presence of the springs which gives the restoring torque, and $T_{f}[\mathrm{~N} \mathrm{~m}]$ represents all friction torques.

The elastic torque, $T_{s}$ in (B.1), is a piecewise linear function of the admissible angles given by

$T_{s}\left(\theta_{t h}\right)= \begin{cases}T_{S_{3}}\left(\theta_{t h}\right) & \text { if } \theta_{t h} \in\left[\theta_{\min } ; \theta_{L H}-\frac{\Delta \theta}{2}\right], \\ 0 & \text { if } \theta_{t h} \in\left[\theta_{L H}-\frac{\Delta \theta}{2} ; \theta_{L H}+\frac{\Delta \theta}{2}\right], \\ T_{S_{1}}\left(\theta_{t h}\right) & \text { if } \theta_{t h} \in\left[\theta_{L H}+\frac{\Delta \theta}{2} ; \theta_{12}\right], \\ T_{S_{2}}\left(\theta_{t h}\right) & \text { if } \theta_{t h} \in\left[\theta_{12} ; \theta_{\max }\right],\end{cases}$

with

$T_{S_{1}}\left(\theta_{t h}\right)=K_{s_{1}}\left[\theta_{t h}-\left(\theta_{L H}+\frac{\Delta \theta}{2}\right)\right]+T_{\text {open }}$,

$T_{S_{2}}\left(\theta_{t h}\right)=K_{S_{2}}\left(\theta_{t h}-\theta_{12}\right)+T_{S_{1}}\left(\theta_{12}\right)$,

$T_{S_{3}}\left(\theta_{t h}\right)=-K_{S_{3}}\left[\left(\theta_{L H}-\frac{\Delta \theta}{2}\right)-\theta_{t h}\right]-T_{\text {close }}$,

where $K_{s_{1}}, K_{s_{2}}, K_{s_{3}}[\mathrm{~N} \mathrm{~m} / \mathrm{deg}]$ are the stiffness coefficients in each region of interest; $T_{\text {close }}[\mathrm{N} \mathrm{m}]$ is the minimum torque necessary to close the valve; $T_{\text {open }}[\mathrm{N} \mathrm{m}]$ is the minimum torque necessary to open the valve; $\theta_{L H}$ [deg] is the limp-home angular position; $\Delta \theta$ [deg] is the clearance between the teeth of the gear, and $\theta_{12}$ [deg] is the discontinuity point of the slope of the elastic torque (see also Fig. B1a and $b$ ).

The model of the friction torque is based on a static Coulomb model modified in order to include the Stribeck effect as (see also Fig. B1c)

$T_{f}\left(\omega_{t h}\right)=\left[T_{c}+\left(T_{s t}-T_{c}\right) e^{-\left|\omega_{t h} / \omega_{s}\right|}\right] \operatorname{sign}\left(\omega_{t h}\right)+\beta_{t h} \frac{\omega_{t h}}{K}$,

where $T_{c}[\mathrm{~N} \mathrm{~m}]$ is the Coulomb friction torque; $T_{s t}[\mathrm{~N} \mathrm{~m}]$ is the stiction friction torque; $\omega_{s}[\mathrm{deg} / \mathrm{s}]$ is the Stribeck velocity, and $\beta_{\text {th }}$ [Nms/rad] is the equivalent linear damping coefficient. 

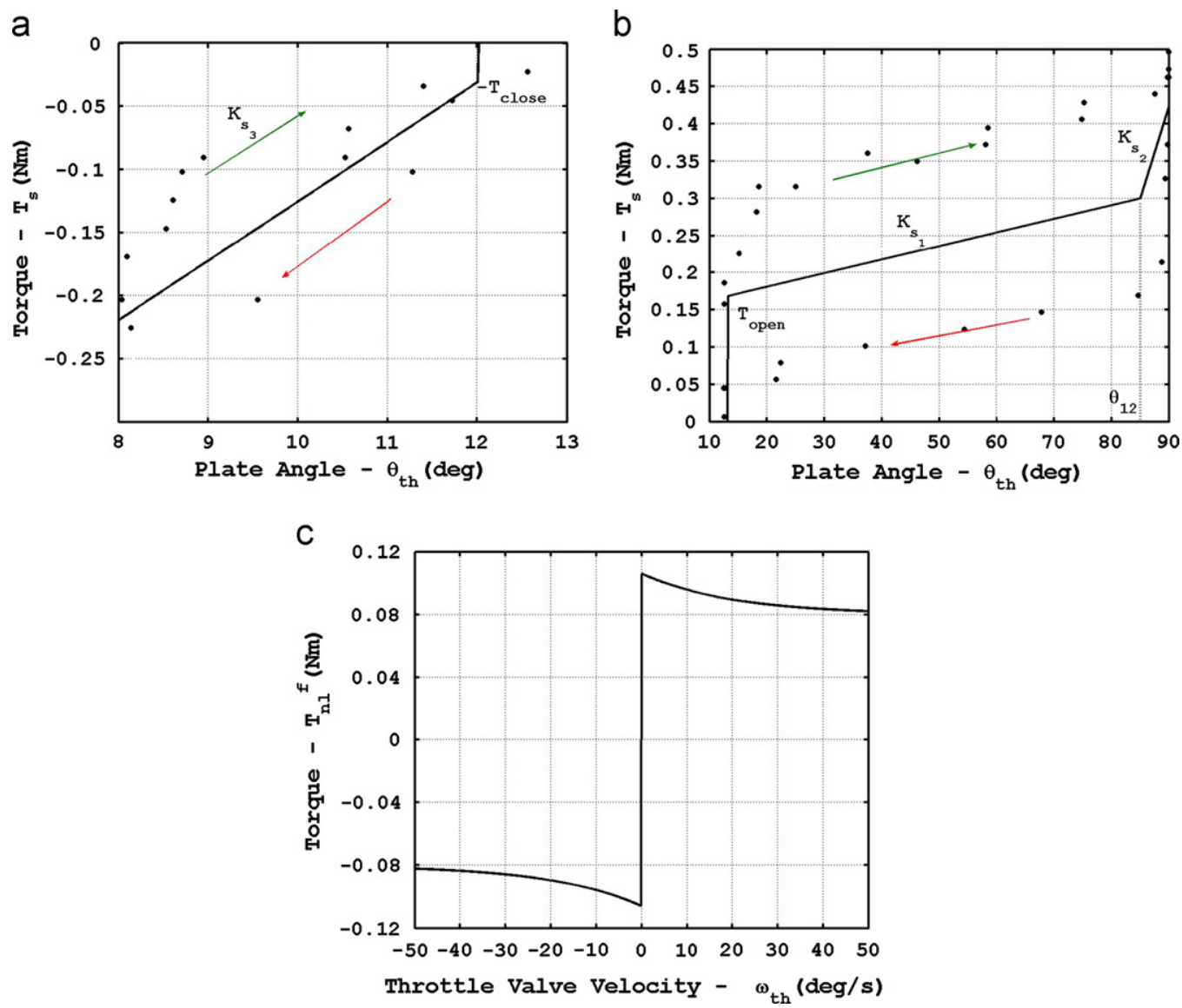

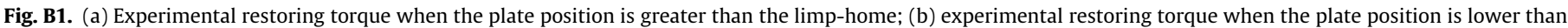
the limp home; (c) nonlinear term of the friction torque based on identified plant parameters.

Further details on model derivation, identification and validation as well as model parameters can be found in di Bernardo, di Gaeta, Montanaro, et al. (2010) and references therein.

Using the setup described in Section 4.1, it has been possible to capture and confirm experimentally the nonlinear behavior exhibited by the throttle body. A notable consequence of friction is the presence of stick-slip behavior as shown in Fig. B2a. When this kind of unwanted dynamics appear, rigid body elements alternatively stick and slip with respect to each other. The combined action of friction and spring torques causes an hysteretic behavior in the system response as confirmed in Fig. B2b.

In what follows it is shown that the ETB model (B.1) can be recast as a linear, time-varying system in canonical form, thus fitting within the class of systems modeled by (1), as required by the MCS strategy. Further details can also be found in di Bernardo, di Gaeta, Montanaro, et al. (2010).

Since the electrical dynamics are faster than the mechanical ones, the former can be neglected, thus yielding:

$$
\left\{\begin{array}{l}
\frac{d \theta_{t h}}{d t}=\omega_{t h}, \\
\frac{d \omega_{t h}}{d t}=\frac{K K_{t}}{J} G_{r} I\left(v_{a}, \omega_{t h}\right)-\frac{K}{J} T_{s}\left(\theta_{t h}\right)-\frac{K}{J} T_{f}\left(\omega_{t h}\right),
\end{array}\right.
$$

where the current $I$ is a static function of the armature voltage $v_{a}$ and angular velocity of the plate $\omega_{t h}$ as

$I=\frac{v_{a}}{R}-\frac{K_{v} G_{r}}{R K} \omega_{t h}$
Let us now rewrite the spring torque as

$T_{s}\left(\theta_{t h}\right)=K_{s_{1}} \theta_{t h}+T_{n l}^{s}\left(\theta_{t h}\right)$,

$T_{n l}^{S}\left(\theta_{t h}\right)= \begin{cases}\Delta T_{S_{3}}\left(\theta_{t h}\right) & \text { if } \theta_{t h} \in\left[\theta_{\min } ; \theta_{L H}-\frac{\Delta \theta}{2}\right], \\ \Delta T_{S_{4}}\left(\theta_{t h}\right) & \text { if } \theta_{t h} \in\left[\theta_{L H}-\frac{\Delta \theta}{2} ; \theta_{L H}+\frac{\Delta \theta}{2}\right], \\ \Delta T_{S_{1}}\left(\theta_{t h}\right) & \text { if } \theta_{t h} \in\left[\theta_{L H}+\frac{\Delta \theta}{2} ; \theta_{12}\right], \\ \Delta T_{S_{2}}\left(\theta_{t h}\right) & \text { if } \theta_{t h} \in\left[\theta_{12} ; \theta_{\max }\right],\end{cases}$

where

$\Delta T_{S_{1}}\left(\theta_{\text {th }}\right)=-K_{S_{1}}\left(\theta_{L H}+\frac{\Delta \theta}{2}\right)+T_{\text {open }}$,

$\Delta T_{S_{2}}\left(\theta_{t h}\right)=\left(K_{S_{2}}-K_{S_{1}}\right)\left(\theta_{t h}-\theta_{12}\right)+\Delta T_{S_{1}}\left(\theta_{t h}\right)$,

$\Delta T_{S_{3}}\left(\theta_{t h}\right)=\left(K_{s_{3}}-K_{S_{1}}\right) \theta_{t h}-K_{S_{3}}\left(\theta_{L H}-\frac{\Delta \theta}{2}\right)-T_{\text {close }}$,

$\Delta T_{S_{4}}\left(\theta_{t h}\right)=-K_{s_{1}} \theta_{t h}$,

and the friction torque as

$T_{f}\left(\omega_{t h}\right)=\beta_{t h} \omega_{t h}+T_{n l}^{f}\left(\omega_{t h}\right)$,

where

$T_{n l}^{f}\left(\omega_{t h}\right)=\left[T_{c}+\left(T_{s t}-T_{c}\right) e^{-\left|\omega_{t h} / \omega_{s}\right|}\right] \operatorname{sign}\left(\omega_{t h}\right)$.

Substituting expressions (B.7), (B.10) in the model (B.5), one gets

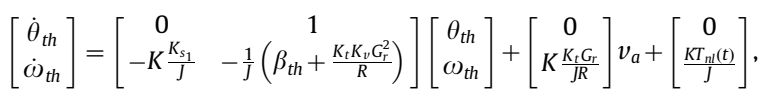



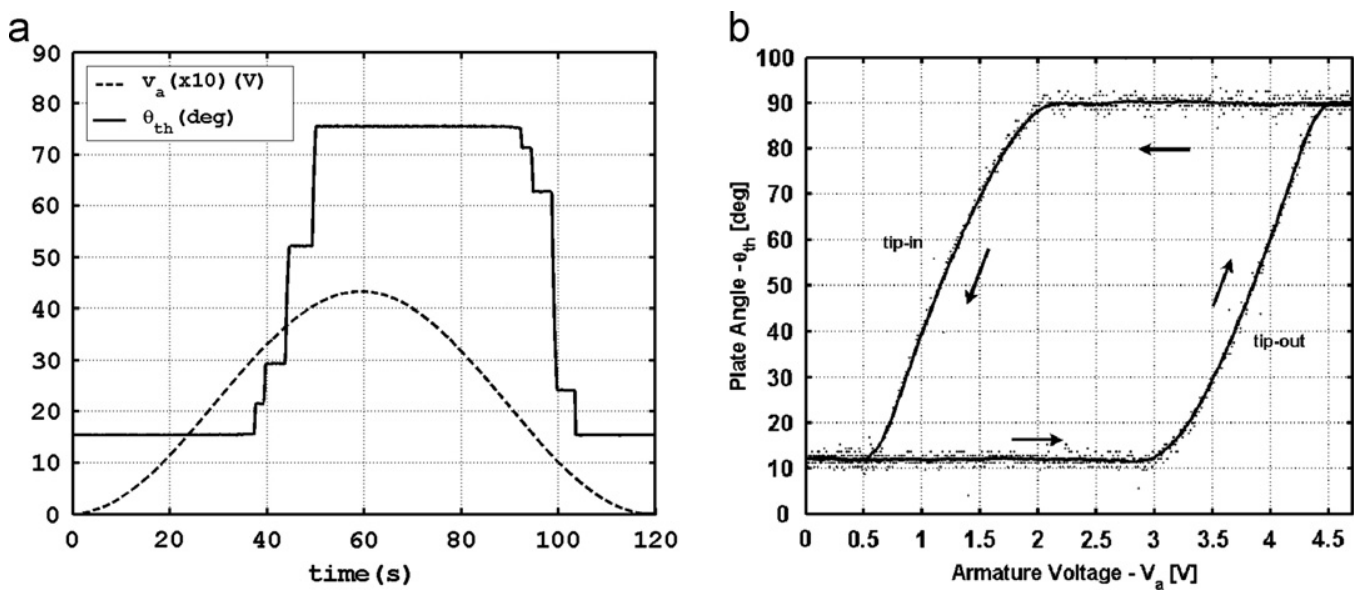

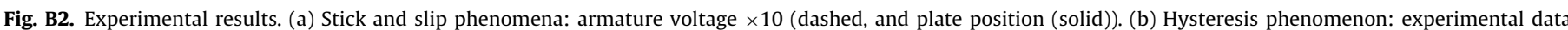
from the plant (dotted) and average data (solid).

with

$T_{n l}(t)=T_{n l}\left(\theta_{t h}(t), \omega_{t h}(t)\right)=T_{n l}^{s}\left(\theta_{t h}(t)\right)+T_{n l}^{f}\left(\omega_{t h}(t)\right)$.

Define now the state and the input vectors as $x=\left[\theta_{t h} \omega_{t h}\right]^{T}$ and $u=v_{a}$, respectively. vector. System (B.12) can be written as

$\dot{x}(t)=A_{0} x(t)+B_{0} u+\eta(t)$,

where

$A_{0}=\left[\begin{array}{cc}0 & 1 \\ -K \frac{K_{s_{1}}}{J} & -\frac{1}{J}\left(\beta_{t h}+\frac{K_{t} K_{v} G_{r}^{2}}{R}\right)\end{array}\right]$,

$B_{0}=\left[\begin{array}{c}0 \\ K \frac{K_{t} G_{r}}{J R}\end{array}\right]$,

$\eta(t)=\left[\begin{array}{c}0 \\ \frac{K}{J} T_{n l}(t)\end{array}\right]$

Then, as pointed out in Section 2, following Stoten and Benchoubane (1990b), and taking into account that $\theta_{\min }>0$, the non-null component of the disturbance vector may be written as

$\frac{K}{J} T_{n l}(t)=\frac{K T_{n l}(t)}{J \theta_{t h}(t)} \theta_{t h}(t)$

and (B.13) takes the form of (1), namely,

$\dot{x}(t)=A(t) x(t)+B b u$,

with

$A(t)=\left[\begin{array}{cc}0 & 1 \\ -a_{1}(t) & -a_{2}(t)\end{array}\right], \quad B=\left[\begin{array}{l}0 \\ 1\end{array}\right]$,

where

$a_{1}(t)=\frac{K}{J}\left[K_{s_{1}}+\frac{T_{n l}(t)}{\theta_{t h}(t)}\right], \quad a_{2}(t)=\frac{1}{J}\left(\beta_{t h}+\frac{K_{t} K_{v} G_{r}^{2}}{R}\right), \quad b=K \frac{K_{t} G_{r}}{J R}$.

\section{References}

Alt, B., Blath, J., Svaricek, F., \& Schultalbers, M. (2010). Self-tuning control design strategy for an electronic throttle with experimental robustness analysis. In Proceedings of the American control conference (pp. 6127-6132). Baltimore, ML USA.

Barić, M., Petrović, I., \& Perić, N. (2005). Neural network-based sliding mode control of electronic throttle. Engineering Applications of Artificial Intelligence, 18, 951-961.

di Bernardo, M., di Gaeta, A., Giglio, V., Montanaro, U., \& Santini, S. (2009). Design and validation of a novel model reference adaptive algorithm to control ETB for drive-by-wire applications. International Journal of Passenger Cars Mechanical Systems, 2, 1268-1284. di Bernardo, M., di Gaeta, A., Montanaro, U., Olm, J. M., \& Santini, S. (2011). Discrete-time MRAC with minimal controller synthesis of an electronic throttle body. In Proceedings of the eighteenth IFAC world congress (pp. 5064-5069) Milan, Italy.

di Bernardo, M., di Gaeta, A., Montanaro, U., \& Santini, S. (2010). Synthesis and experimental validation of the novel LQ-NEMCSI adaptive strategy on an electronic throttle valve. IEEE Transactions on Control Systems Technology, 18 1325-1337.

di Bernardo, M., di Gennaro, F., Olm, J. M., \& Santini, S. (2010). Discrete-time minimal control synthesis adaptive algorithm. International Journal of Control 83, 2641-2657.

di Bernardo, M., Montanaro, U., Olm, J.M., \& Santini, S. Model reference adaptive control of discrete-time piecewise linear systems. International Journal of Robust and Nonlinear Control, http://dx.doi.org/10.1002/rnc.2786, in press.

di Bernardo, M. Montanaro, U., \& Santini, S. (2007). Novel hybrid MRAC-LQ contro schemes: Synthesis, analysis and applications. International Journal of Control, 80, 940-961.

di Bernardo, M., Montanaro, U., \& Santini, S. (2008). Novel switched model reference adaptive control for continuous piecewise affine systems. In 47th IEEE Conference on Decision and Control (pp. 1925-1930).

di Bernardo, M., Montanaro, U., Santini, S. (2009). Hybrid minimal control synthesis identification of continuous piecewise linear systems. In 48th IEEE conference on decision and control (pp. 3188-3193).

di Bernardo, M., Montanaro, U., \& Santini, S. (2010a). Identification of hybrid bimodal piecewise affine systems via minimal control synthesis adaptive control. In Eighth IFAC symposium on nonlinear control systems (pp. 356-361).

di Bernardo, M., Montanaro, U., \& Santini, S. (2010b). Minimal control synthesis adaptive control of continuous bimodal piecewise affine systems. SIAM Journa on Control and Optimization, 48, 4242-4261.

di Bernardo, M., Montanaro, U., \& Santini, S. (2011). Canonical forms of generic piecewise linear continuous systems. IEEE Transactions on Automatic Control 56, 1911-1915.

di-Bernardo, M., Montanaro, U., \& Santini, S. (2013). Hybrid model reference adaptive control of piecewise affine systems. IEEE Transactions on Automatic Control, 58, 304-316.

di Bernardo, M., Montanaro, U., Santini, S., \& Hoyos Velasco, C.I. (2010). Experimental validation of a novel adaptive controller for piecewise affine systems. In IEEE international symposium on circuits and systems (pp. 1543-1546).

di Bernardo, M., Hoyos Velasco, C. I., Montanaro, U., \& Santini, S. (2012). Experimental implementation and validation of a novel minimal control synthesis adaptive controller for continuous bimodal piecewise affine systems. Control Engineering Practice, 20, 269-281.

Bursi, O. S., Stoten, D. P., Tondini, N., \& Vulcan, L. (2009). Stability and accuracy analysis of a discrete model reference adaptive controller without and with time delay. International Journal of Numerical Methods in Engineering, 82, 1158-1179.

Bursi, O. S., Stoten, D. P., \& Vulcan, L. (2007). Convergence and frequency-domain analysis of a discrete first-order model reference adaptive controller. Structural Control and Health Monitoring, 14, 777-807.

Corno, M., Tanelli, M., Savaresi, S., \& Fabbri, L. (2011). Design and validation of a gain-scheduled controller for the electronic throttle body in ride-by-wire racing motorcycles. IEEE Transactions on Control Systems Technology, 19, 18-30.

Dagci, O. H., Pan, Y., \& Ozguner, U. (2002). Sliding mode control of electronic throttle valve. In Proceedings of the American control conference (pp. 1996-2001). Anchorage, AK, USA.

Deur, J., Pavkovic, D., Peric, N., Janz, M., \& Hrovat, D. (2004). An electronic throttle control strategy including compensation of friction and limp-home effects. IEEE Transactions on Industry Applications, 40, 821-834. 
di Gaeta, A., Montanaro, U., \& Giglio, V. (2010). Idle speed control of GDI-SI engines via ECU-1D engine co-simulation. SAE Technical Paper, 2010-01-2220.

di Gaeta, A., Montanaro, U., \& Giglio, V. (2011). Model-based control of the air fue ratio for gasoline direct injection engines via advanced co-simulation: An approach to reduce the development cycle of engine control systems. Journal of Dynamic Systems, Measurement, and Control, 133, 061006.

Gizatullin, A. O., \& Edge, K. A. (2007). Adaptive control for a multi-axis hydraulic test rig. Proceedings of the Institution of Mechanical Engineers - Part I: Journal of Systems and Control Engineering, 221, 183-198.

Landau, Y. D. (1979). Adaptive control: The model reference approach. NY: Marcel Dekker Inc.

Montanaro, U., di Gaeta, A., \& Giglio, V. (2011). An MRAC approach for tracking and ripple attenuation of the common rail pressure for GDI engines. In Proceedings of the eighteenth IFAC world congress (pp. 4173 - 4180). Milan, Italy.

Ozguner, U., Hong, S., \& Pan, Y. (2001). Discrete-time sliding mode control of electronic throttle valve. In Proceedings of the fortieth IEEE conference on decision and control (pp. 1819-1824). Newblock Orlando, FL, USA.

Pan, Y., Dagci, O., \& Ozguner, U. (2001). Variable structure control of electronic throttle valve. In IEEE vehicle electronics conference (pp. 103-108). Tottori, Japan.

Pavkovic, D., Deur, J., Jansz, M., \& Peric, N. (2003). Self-tuning control of an electronic throttle. In Proceedings of 2003 IEEE conference on control applications (pp. 149-154). Istanbul, Turkey.

Pavković, D., Deur, J., Jansz, M., \& Perić, N. (2006). Adaptive control of automotive electronic throttle. Control Engineering Practice, 14, 121-136.

Popov, V. M. (1973). Hyperstability of automatic control systems. NY: Springer.

Rossi, C., Tilli, A., \& Tonielli, A. (2000). Robust control of a throttle body for drive by wire operation of automotive engines. IEEE Transactions on Control Systems Technology, 8, 993-1002.

Rossi, L., Irace, A., Montanaro, U., di Bernardo, M., \& Breglio, G. (2010). Structural vibration control of a cantilever beam by MRAC method. In Second international symposium on reliability of optoelectronics for space.
Rugh, W. J. (1996). Linear system theory (2nd ed.). Upper Saddle River, NJ: PrenticeHall.

Stoten, D. P., \& Benchoubane, H. (1990a). Empirical studies of an MRAC algorithm with minimal controller synthesis. International Journal of Control, 51, 823-849.

Stoten, D. P., \& Benchoubane, H. (1990b). Robustness of a minimal controller synthesis algorithm. International Journal of Control, 51, 851-861.

Stoten, D. P., \& di Bernardo, M. (1996). Application of the minimal control synthesis algorithm to the control and synchronization of chaotic systems. International Journal of Control, 65, 925-938.

Stoten, D. P., \& Gómez, E. G. (2001). Adaptive control of shaking tables using the minimal controller synthesis algorithm. Philosophical Transactions of the Royal Society of London, 357, 1697-1723.

Vasak, M., Baoti, M., Morari, M., Petrovic, I., \& Peric, N. (2006). Constrained optimal control of an electronic throttle. International Journal of Control, 79, 465-478.

Vasak, M., Baotic, M., Petrovic, I., \& Peric, N. (2004). Electronic throttle state estimation and hybrid theory based optimal control. In Proceedings of the IEEE international symposium on industrial electronics (pp. 323-328). Ajaccio, France.

Vasak, M., Baoti, M., Petrovic, I., \& Peric, N. (2007). Hybrid theory-based timeoptimal control of an electronic throttle. IEEE Transactions on Industrial Electronics, 54, 1483-1494.

Vasak, M., Petrovic, I., \& Peric, N. (2003). State estimation of an electronic throttle body. In Proceedings of the IEEE international conference on industrial technology (pp. 472-477, Vol. 1).

de Witt, C. C., Kolmanovsky, I., \& Sun, J. (2001). Adaptive pulse control of electronic throttle. In Proceedings of the American control conference (pp. 2872-2877). Arlington, VI, USA.

de Witt, C. C., Olsson, H., Astrom, K. J., \& Lischinsky, P. (1995). A new model for control of systems with friction. IEEE Transactions on Automatic Control, 40, 419-425.

Yokoyama, M., Shimizu, K., \& Okamoto, N. (1998). Application of sliding-mode servo controllers to electronic throttle control. In Proceedings of the 37th IEEE conference on decision and control (pp. 1541-1545). Tampa, FL, USA 